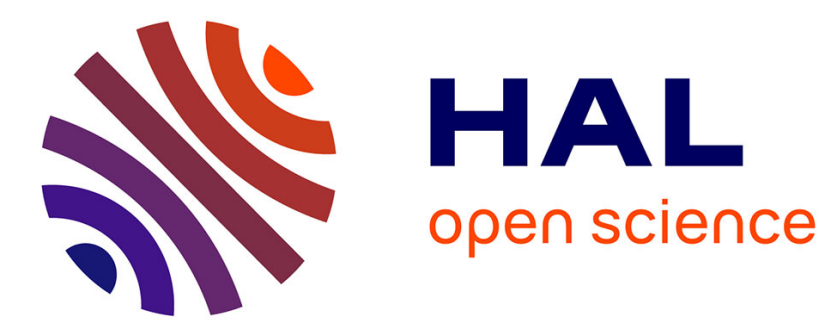

\title{
Topological Hopf algebras, quantum groups and deformation quantization
}

Philippe Bonneau, Daniel Sternheimer

\section{To cite this version:}

Philippe Bonneau, Daniel Sternheimer. Topological Hopf algebras, quantum groups and deformation quantization. 2003. hal-00000507

\section{HAL Id: hal-00000507 \\ https://hal.science/hal-00000507}

Preprint submitted on 21 Jul 2003

HAL is a multi-disciplinary open access archive for the deposit and dissemination of scientific research documents, whether they are published or not. The documents may come from teaching and research institutions in France or abroad, or from public or private research centers.
L'archive ouverte pluridisciplinaire HAL, est destinée au dépôt et à la diffusion de documents scientifiques de niveau recherche, publiés ou non, émanant des établissements d'enseignement et de recherche français ou étrangers, des laboratoires publics ou privés. 


\title{
Topological Hopf algebras, quantum groups and deformation quantization
}

\author{
Philippe Bonneau and Daniel Sternheimer \\ Laboratoire Gevrey de Mathématique physique, Université de Bourgogne, \\ BP 47870, F-21078 Dijon Cedex, France. \\ Philippe.Bonneau@u-bourgogne.fr, Daniel.Sternheimer@u-bourgogne.fr
}

January 5, 2003; revised May 7, 2003

\begin{abstract}
After a presentation of the context and a brief reminder of deformation quantization, we indicate how the introduction of natural topological vector space topologies on Hopf algebras associated with Poisson Lie groups, Lie bialgebras and their doubles explains their dualities and provides a comprehensive framework. Relations with deformation quantization and applications to the deformation quantization of symmetric spaces are described.
\end{abstract}

Mathematics Subject Classifcations (2000): Primary 54C40, 14E20; Secondary 46E25, 20C20

Keywords: Hopf algebras, topological vector spaces, quantum groups, deformation quantization.

\section{Introduction}

\subsection{Presentation of the context.}

The expression "quantum groups" is a name coined by Drinfeld (see [Dri87]) in the frst half of the 80's which is superb, even if the notion is not necessarily quantum and the objects are not really groups. But they are Hopf algebras and their theory can be viewed as an avatar of deformation quantization [BFFLS] (see [DS02] for a recent review which this presentation complements), applied to the quantization of Poisson-Lie groups.

The philosophy underlying the role of deformations in physics has been consistently put forward by Flato, almost since the defnition of the deformation of rings and algebras by Gerstenhaber [Ger64], and was eventually expressed by him in [Fla82]. In short, the passage from one level of physical theory to another, when a new fundamental constant is imposed by experiments, can be understood (and might even have been predicted) using deformation theory. The only question is, in which category do we seek for deformations? Usually physics is rather conservative and if we start e.g. with the category of associative or Lie algebras, we tend to deform in the same category.

But there are important instances of generalizations of this principle. The most elaborate is maybe noncommutative geometry, where the strategy is to formulate the "undeformed" (commutative) geometry in terms of algebraic structures in such a way that it becomes possible to "plug in" the deformation (noncommutativity) in a quite natu$\mathrm{ral}$, and mathematically rigorous, manner. We shall not elaborate on that aspect here, refering e.g. to [Co00] for a presentation, to [CDV02] for important recent examples of noncommutative manifolds, and to [Co94, [CFS92] for the basics and a relation with deformation quantization.

We shall concentrate on another prominent example: quantum groups. Instead of looking at the associative algebra of functions over a Poisson-Lie group or at the enveloping algebra, one makes full use of the Hopf algebra structure in both cases. In general both the product and the coproduct have to be (compatibly) deformed, but cohomological results (Dri89] and section 3.1) show that, when the Lie group is semi-simple, the deformation is always equivalent to a "preferred" one, that is, a deformation where only the product or the coproduct (resp.) is deformed. The group aspect is a special case of deformation quantization and we shall show that the enveloping algebra aspect can be seen as its dual, in the sense of topological vector spaces duality.

\subsection{Deformation theory of algebras.}

A concise formulation of a Gerstenhaber deformation of an algebra (associative, Lie, bialgebra, etc.) is [Ger64, BFGP94: 
Defnition 1 A deformation of an algebra A over a £eld $\mathbb{K}$ is a $\mathbb{K}[[v]]$-algebra $\tilde{A}$ such that $\tilde{A} / v \tilde{A} \approx A$. Two deformations $\tilde{A}$ and $\tilde{A}^{\prime}$ are said equivalent if they are isomorphic over $\mathbb{K}[[\boldsymbol{V}]]$ and $\tilde{A}$ is said trivial if it is isomorphic to the original algebra A considered by base £eld extension as a $\mathbb{K}[[v]]$-algebra.

Whenever we consider a topology on $A, \tilde{A}$ is supposed to be topologically free. For associative (resp. Lie) algebras, Defnition 1 tells us that there exists a new product $*($ resp. bracket $[\cdot, \cdot]$ ) such that the new (deformed) algebra is again associative (resp. Lie). Denoting the original composition laws by ordinary product (resp. $\{\cdot, \cdot\}$ ) this means that, for $u, v \in A$ (we can extend this to $A[[v]]$ by $\mathbb{K}[[v]]$-linearity) we have:

$$
\begin{aligned}
& u * v=u v+\sum_{r=1}^{\infty} v^{r} C_{r}(u, v) \\
& {[u, v]=\{u, v\}+\sum_{r=1}^{\infty} v^{r} B_{r}(u, v)}
\end{aligned}
$$

where the $C_{r}$ are Hochschild 2-cochains and the $B_{r}$ (skew-symmetric) Chevalley 2-cochains, such that for $u, v, w \in A$ we have $(u * v) * w=u *(v * w)$ and $\mathscr{S}[[u, v], w]=0$, where $\mathscr{S}$ denotes summation over cyclic permutations.

For a (topological) bialgebra (an associative algebra $A$ where we have in addition a coproduct $\Delta: A \longrightarrow A \otimes A$ and the obvious compatibility relations), denoting by $\otimes_{v}$ the tensor product of $\mathbb{K}[[v]]$-modules, we can identify $\tilde{A} \hat{\otimes}_{v} \tilde{A}$ with $(A \hat{\otimes} A)[[v]]$, where $\hat{\otimes}$ denotes the algebraic tensor product completed with respect to some topology (e.g. projective for Fréchet nuclear topology on A), we similarly have a deformed coproduct $\tilde{\Delta}=\Delta+\sum_{r=1}^{\infty} v^{r} D_{r}$, $D_{r} \in \mathscr{L}(A, A \hat{\otimes} A)$, satisfying $\tilde{\Delta}(u * v)=\tilde{\Delta}(u) * \tilde{\Delta}(v)$. In this context appropriate cohomologies can be introduced [GS90, Bon92]. There are natural additional requirements for Hopf algebras.

Equivalence means that there is an isomorphism $T_{v}=I+\sum_{r=1}^{\infty} v^{r} T_{r}, T_{r} \in \mathscr{L}(A, A)$ so that $T_{v}\left(u *^{\prime} v\right)=\left(T_{v} u *\right.$ $\left.T_{v} v\right)$ in the associative case, denoting by $*$ (resp. $\left.*^{\prime}\right)$ the deformed laws in $\tilde{A}$ (resp. $\tilde{A}^{\prime}$ ); and similarly in the Lie, bialgebra and Hopf cases. In particular we see (for $r=1$ ) that a deformation is trivial at order 1 if it starts with a 2-cocycle which is a 2-coboundary. More generally, exactly as above, we can show [BFFLS] ([GS90, Bon92] in the Hopf case) that if two deformations are equivalent up to some order $t$, the condition to extend the equivalence one step further is that a 2-cocycle (defned using the $T_{k}, k \leq t$ ) is the coboundary of the required $T_{t+1}$ and therefore the obstructions to equivalence lie in the 2-cohomology. In particular, if that space is null, all deformations are trivial.

Unit. An important property is that a deformation of an associative algebra with unit (what is called a unital algebra) is again unital, and equivalent to a deformation with the same unit. This follows from a more general result of Gerstenhaber (for deformations leaving unchanged a subalgebra) and a proof can be found in [GS88].

Remark 1 In the case of (topological) bialgebras or Hopf algebras, equivalence of deformations has to be understood as an isomorphism of (topological) $\mathbb{K}[[v]]$-algebras, the isomorphism starting with the identity for the degree 0 in $v$. A deformation is again said trivial if it is equivalent to that obtained by base £eld extension. For Hopf algebras the deformed algebras may be taken (by equivalence) to have the same unit and counit, but in general not the same antipode.

\subsection{Deformation quantization and physics.}

Intuitively, classical mechanics is the limit of quantum mechanics when $\hbar=\frac{h}{2 \pi}$ goes to zero. But how can this be realized when in classical mechanics the observables are functions over phase space (a Poisson manifold) and not operators? The deformation philosophy promoted by Flato shows the way: one has to look for deformations of algebras of classical observables, functions over Poisson manifolds, and realize there quantum mechanics in an autonomous manner.

What we call "deformation quantization" relates to (and generalizes) what in the conventional (operatorial) formulation are the Heisenberg picture and Weyl's quantization procedure. In the latter Wey31], starting with a classical observable $u(p, q)$, some function on phase space $\mathbb{R}^{2 \ell}$ (with $p, q \in \mathbb{R}^{\ell}$ ), one associates an operator (the corresponding quantum observable) $\Omega(u)$ in the Hilbert space $L^{2}\left(\mathbb{R}^{\ell}\right)$ by the following general recipe:

$$
u \mapsto \Omega_{w}(u)=\int_{\mathbb{R}^{2 \ell}} \tilde{u}(\xi, \eta) \exp (i(P . \xi+Q . \eta) / \hbar) w(\xi, \eta) d^{\ell} \xi d^{\ell} \eta
$$

where $\tilde{u}$ is the inverse Fourier transform of $u, P_{\alpha}$ and $Q_{\alpha}$ are operators satisfying the canonical commutation relations $\left[P_{\alpha}, Q_{\beta}\right]=i \hbar \delta_{\alpha \beta}(\alpha, \beta=1, \ldots, \ell), w$ is a weight function and the integral is taken in the weak operator topology. What is called in physics normal (or antinormal) ordering corresponds to choosing for weight 
$w(\xi, \eta)=\exp \left(-\frac{1}{4}\left(\xi^{2} \pm \eta^{2}\right)\right)$. Standard ordering (the case of the usual pseudodifferential operators in mathematics) corresponds to $w(\xi, \eta)=\exp \left(-\frac{i}{2} \xi \eta\right)$ and the original Weyl (symmetric) ordering to $w=1$. An inverse formula was found shortly afterwards by Eugene Wigner [Wig32] and maps an operator into what mathematicians call its symbol by a kind of trace formula. For example $\Omega_{1}$ de£nes an isomorphism of Hilbert spaces between $L^{2}\left(\mathbb{R}^{2 \ell}\right)$ and Hilbert-Schmidt operators on $L^{2}\left(\mathbb{R}^{\ell}\right)$ with inverse given by

$$
u=(2 \pi \hbar)^{-\ell} \operatorname{Tr}\left[\Omega_{1}(u) \exp ((\xi . P+\eta \cdot Q) / i \hbar)\right]
$$

and if $\Omega_{1}(u)$ is of trace class one has $\operatorname{Tr}\left(\Omega_{1}(u)\right)=(2 \pi \hbar)^{-\ell} \int u \omega^{\ell} \equiv \operatorname{Tr}_{M}(u)$, the "Moyal trace", where $\omega^{\ell}$ is the (symplectic) volume $d x$ on $\mathbb{R}^{2 \ell}$. Looking for a direct expression for the symbol of a quantum commutator, Moyal found Moy49, what is now called the Moyal bracket:

$$
M\left(u_{1}, u_{2}\right)=v^{-1} \sinh (v P)\left(u_{1}, u_{2}\right)=P\left(u_{1}, u_{2}\right)+\sum_{r=1}^{\infty} \frac{v^{2 r}}{(2 r+1) !} P^{2 r+1}\left(u_{1}, u_{2}\right)
$$

where $2 v=i \hbar, P^{r}\left(u_{1}, u_{2}\right)=\Lambda^{i_{1} j_{1}} \ldots \Lambda^{i_{r} j_{r}}\left(\partial_{i_{1} \ldots i_{r}} u_{1}\right)\left(\partial_{j_{1} \ldots j_{r}} u_{2}\right)$ is the $r^{\text {th }}$ power $(r \geq 1)$ of the Poisson bracket bidifferential operator $P, i_{k}, j_{k}=1, \ldots, 2 \ell, k=1, \ldots, r$ and $\left(\Lambda^{i_{k} j_{k}}\right)=\left(\begin{array}{c}0-I \\ I 0\end{array}\right)$. To $£ \mathrm{x}$ ideas we may assume here $u_{1}, u_{2} \in \mathscr{C}^{\infty}\left(\mathbb{R}^{2 \ell}\right)$ and the sum is taken as a formal series. A corresponding formula for the symbol of a product $\Omega_{1}(u) \Omega_{1}(v)$ can be found in [Gre46], and may now be written more clearly as a (Moyal) star product:

$$
u_{1} *_{M} u_{2}=\exp (v P)\left(u_{1}, u_{2}\right)=u_{1} u_{2}+\sum_{r=1}^{\infty} \frac{v^{r}}{r !} P^{r}\left(u_{1}, u_{2}\right)
$$

The formal series may be deduced (see e.g. [Bie00]) from an integral formula of the type:

$$
\left(u_{1} * u_{2}\right)(x)=c_{\hbar} \int_{\mathbb{R}^{2 \ell} \times \mathbb{R}^{2 \ell}} u_{1}(x+y) u_{2}(x+z) e^{-\frac{i}{\hbar} \Lambda^{-1}(y, z)} d y d z .
$$

It was noticed, however after deformation quantization was introduced, that the composition of symbols of pseudodifferential operators (ordered, like differential operators, "£rst $q$, then $p$ ") is a star product.

One recognizes in (6) a special case of (1), and similarly for the bracket. So, via a Weyl quantization map, the algebra of quantized observables can be viewed as a deformation of that of classical observables.

But the deformation philosophy tells us more. Deformation quantization is not merely "a reformulation of quantizing a mechanical system" [DN01], e.g. in the framework of Weyl quantization: The process of quantization itself is a deformation. In order to show that explicitly it was necessary to treat in an autonomous manner signifcant physical examples, without recourse to the traditional operatorial formulation of quantum mechanics. That was achieved in [BFFLS] with the paradigm of the harmonic oscillator and more, including the angular momentum and the hydrogen atom. In particular what plays here the role of the unitary time evolution operator of a quantized system is the "star exponential" of its classical Hamiltonian $H$ (expressed as a usual exponential series but with "star powers" of $t H / i \hbar, t$ being the time, and computed as a distribution both in phase space variables and in time); in a very natural manner, the spectrum of the quantum operator corresponding to $H$ is the support of the Fourier-Stieltjes transform (in $t$ ) of the star exponential (what Laurent Schwartz had called the spectrum of that distribution). Further examples were (and are still being) developed, in particular in the direction of £eld theory.

That aspect of deformation theory has since 25 years or so been extended considerably. It now includes general symplectic and Poisson (£nite dimensional) manifolds, with further results for in£nite dimensional manifolds, for "manifolds with singularities" and for algebraic varieties, and has many far reaching ramifcations in both mathematics and physics (see e.g. a brief overview in [DS02]). As in quantization itself [Wey31], symmetries (group theory) play a special role and an autonomous theory of star representations of Lie groups was developed, in the nilpotent and solvable cases of course (due to the importance of the orbit method there), but also in signi£cant other examples. The presentation that follows can be seen as an extension of the latter, when one makes full use of the Hopf algebra structures and of the "duality" between the group structure and the set of its irreducible representations.

Finally one should mention that deformation theory and Hopf algebras are seminal in a variety of problems ranging from theoretical physics (see e.g. [CK99, DS02]), including renormalization and Feynman integrals and diagrams, to algebraic geometry and number theory (see e.g. [Ko01, KZ01]), including algebraic curves ála Zagier (cf. [CM03] and Connes' lectures at Collége de France, January to March 2003). 


\section{Some topological Hopf algebras}

We shall now brieay review applications of the deformation theory of algebras in the context of Hopf algebras endowed with appropriate topologies and in the spirit of deformation quantization. That is, we shall consider Hopf algebras of functions on Poisson-Lie groups (or their topological duals) and their deformations, and show how this framework is a powerful tool to understand the standard examples of quantum groups, and more. In order to do so we £rst recall some notions on topological vector spaces and apply them to our context.

\subsection{Well-behaved Hopf algebras}

De£nition 2 A topological vector space (tvs) $V$ is said well-behaved if $V$ is either nuclear and Fréchet, or nuclear and dual of Fréchet [Grt55, Tré67].

Proposition 1 If $V$ is a well-behaved tvs and $W$ a tvs, then

$$
\text { (i) } V^{* *} \simeq V \quad(\text { ii })(V \hat{\otimes} V)^{*} \simeq V^{*} \hat{\otimes} V^{*} \quad \text { (iii) } \operatorname{Hom}_{\mathbb{K}}(V, W) \simeq V^{*} \hat{\otimes} W
$$

where $V^{*}$ denotes the strong topological dual of $V, \hat{\otimes}$ the projective topological tensor product and the base £eld $\mathbb{K}$ is $\mathbb{R}$ or $\mathbb{C}$.

De£nition $3(A, \mu, \eta, \Delta, \varepsilon, S)$ is a WB (well-behaved) Hopf algebra [BFGP94] if

- A is a well-behaved topological vector space.

- The multiplication $\mu: A \hat{\otimes} A \rightarrow A$, the coproduct $\Delta: A \rightarrow A \hat{\otimes} A$, the unit $\eta$, the counit $\varepsilon$, and the antipode $S$ are continuous.

- $\mu, \eta, \Delta, \varepsilon$ and $S$ satisfy the usual axioms of a Hopf algebra.

Corollary 1 If $(A, \mu, \eta, \Delta, \varepsilon, S)$ is a WB Hopf algebra, then $\left(A^{*},{ }^{t} \Delta,{ }^{t} \varepsilon,{ }^{t} \mu,{ }^{t} \eta,{ }^{t} S\right)$ is also a WB Hopf algebra.

\subsection{Examples of well-behaved Hopf algebras [BFGP94]}

Let $G$ be a semi-simple Lie group and $\mathfrak{g}$ its complexi£ed Lie algebra. For simplicity we shall assume here $G$ linear (i.e. with a faithful £nite dimensional representation) but the same results hold, with some modi£cation in the proofs, for any semi-simple Lie group.

\subsubsection{Example 1}

$\mathscr{C}^{\infty}(G)$, the algebra of the smooth functions on $G$, is a WB Hopf algebra (Fréchet and nuclear).

\subsubsection{Example 2}

$\mathscr{D}(G)=\mathscr{C}^{\infty}(G)^{*}$, the algebra of the compactly supported distributions on $G$, is a WB Hopf algebra (dual of Fréchet and nuclear). The product is the transposed map of the coproduct of $\mathscr{C}^{\infty}(G)$ that is, the convolution of distributions.

\subsubsection{Example 3}

$\mathscr{H}(G)$, the algebra of coef£cient functions of £nite dimensional representations of $G$ (or polynomial functions on $G)$ is a WB Hopf algebra, the Hopf structure being that induced from $\mathscr{C}^{\infty}(G)$.

A short description of that algebra is as follows: We take a set $\hat{G}$ of irreducible $£$ nite dimensional representations of $G$ such that there is one and only one element for each equivalence class, and, if $\pi \in \hat{G}$, its contragredient $\pi$ is also in $\hat{G}$. We de£ne $C_{\pi}=\operatorname{vect}\{$ coeffcient functions of $\pi\} \stackrel{\text { Burnside }}{\simeq} \operatorname{End}\left(V_{\pi}\right)$ for $\pi \in \hat{G}$. Then $\mathscr{H}(G) \stackrel{\text { alg. }}{\simeq} \bigoplus_{\pi \in \hat{G}} C_{\pi} \stackrel{\text { v.s. }}{\simeq} \bigoplus_{\pi \in \hat{G}} \operatorname{End}\left(V_{\pi}\right)$. So we take on $\mathscr{H}(G)$ the "direct sum" topology of $\bigoplus_{\pi \in \hat{G}} \operatorname{End}\left(V_{\pi}\right)$. Then $\mathscr{H}(G)$ is dual of Fréchet and nuclear, that is, WB. 


\subsubsection{Example 4}

Let $\mathscr{A}(G)$, the algebra of "generalized distributions", be de£ned by $\mathscr{A}(G)=\mathscr{H}(G)^{*} \stackrel{\text { alg. }}{\simeq} \prod_{\pi \in \hat{G}} \operatorname{End}\left(V_{\pi}\right)$. The (product) topology is Fréchet and nuclear, and therefore $\mathscr{A}(G)$ is WB.

\subsection{Inclusions [BP96, BFGP94]}

We denote by $\cup \mathfrak{g}$ the universal enveloping algebra of $\mathfrak{g}$ and by $\mathbb{C} G$ the group algebra of $G$. All the following inclusions are inclusions of Hopf algebras. $\Subset, \ni, \uplus, \cap$ mean a dense inclusion.

$$
\begin{array}{ccccc|cc}
\cup \mathfrak{g} & \Subset & \mathscr{A}(G) & \ni & \mathbb{C} G & \mathscr{H}(G) & \\
& & \uplus & & & \mathbb{C} & (*) \\
\mathrm{Ug} & \subset & \mathscr{D}(G) & \ni & \mathbb{C} G & \mathscr{C}^{\infty}(G) &
\end{array}
$$

$(*)$ is true if and only if $G$ is linear, but comparable results can be obtained for $G$ non linear.

\section{Topological quantum groups}

We shall now deform the preceding topological Hopf algebras and indicate how this explains various models of quantum groups. For clarity of the exposition, throughout this Section and the remainder of the paper, we shall limit to a minimum the details concerning the Hopf algebra structures other than product and coproduct. But whenever we write Hopf algebras and not only bialgebras, the relevant structures are included in the discussion and dealing with them is quite straightforward.

\subsection{Quantization}

Theorem 1 ([Dri89]) Let $\mathfrak{g}$ be a semi-simple Lie algebra and $\left(\mathrm{U} \mathfrak{g}, \mu_{0}, \Delta_{0}\right)$ denote the usual Hopf structure on $\cup \mathfrak{g}$.

1. If $\left(\mathrm{U}_{t} \mathfrak{g}, \mu_{t}\right)$ is a deformation (as an algebra) of $\left(\mathrm{U} \mathfrak{g}[[t]], \mu_{0}\right)$ then $\mathrm{U}_{t} \mathfrak{g} \stackrel{\varphi}{\simeq} \cup \mathfrak{g}[[t]]$ (i.e. $\cup \mathfrak{g}$ is rigid).

2. If $\left(\mathrm{U} \mathfrak{g}[[t]], \mu_{0}, \Delta_{t}\right)$ is a deformation (as a Hopf algebra) of $\left(\mathrm{U} \mathfrak{g}[[t]], \mu_{0}, \Delta_{0}\right)$ then

$$
\exists P_{t} \in(\mathrm{Ug} \otimes \mathrm{Ug})[[t]] \text { such that } P_{t=0}=\mathrm{Id} \text { and } \Delta_{t}(a)=P_{t} . \Delta_{0}(a) . P_{t}^{-1}, \forall a \in \mathrm{Ug} \text {. }
$$

An isomorphism $\varphi$ (it is not unique!) appearing in item 1 above is called a Drinfeld isomorphism.

Corollary 2 ([BFGP94) Let $G$ be a linear semi-simple Lie group and $\mathfrak{g}$ be its complexifed Lie algebra.

1. If $\mathrm{U}_{t} \mathfrak{g}$ is a deformation of $\mathrm{U} \mathfrak{g}$ (a "quantum group") then $\left(\mathrm{U}_{t} \mathfrak{g}, \mu_{t}, \Delta_{t}\right) \simeq\left(\mathrm{U} \mathfrak{g}[[t]], \mu_{0}, P_{t} \Delta_{0} P_{t}^{-1}\right)$.

2. $\mathscr{A}_{t}(G):=\left(\mathscr{A}(G)[[t]], \mu_{0}, P_{t} \cdot \Delta_{0} \cdot P_{t}^{-1}\right)$ is a Hopf deformation of $\mathscr{A}(G)$ and $\mathrm{U}_{t} \mathfrak{g} \stackrel{\text { Hopf }}{\subset} \mathscr{A}_{t}(G)$.

3. $\mathscr{D}_{t}(G):=\left(\mathscr{D}(G)[[t]], \mu_{0}, P_{t} \cdot \Delta_{0} \cdot P_{t}^{-1}\right)$ is a Hopf deformation of $\mathscr{D}(G)$ and $\mathrm{U}_{t} \mathfrak{g}{ }^{\text {Hopf }} \mathscr{D}_{t}(G)$.

4. $\mathscr{C}_{t}^{\infty}(G):=\mathscr{D}_{t}(G)^{*}$ and $\mathscr{H}_{t}(G):=\mathscr{A}_{t}(G)^{*}$ are quantized algebras of functions. They are Hopf deformations of $\mathscr{C}^{\infty}(G)$ and $\mathscr{H}(G)$.

Similar results hold in the non linear case [BP96] and for other WB Hopf algebras (e.g. constructed with infnite dimensional representations) [Bid96].

Proof. (1) Direct consequence of Theorem 11. (2) $P_{t} \in(\mathrm{Ug} \otimes U \mathfrak{g})[[t]] \subset(\mathscr{A}(G) \hat{\otimes} \mathscr{A}(G))[[t]]$. We obtain coassociativity from $\cup_{\mathfrak{g}} \Subset \mathscr{A}(G)$. (3) By restriction of (2). (4) By simple dualization from (2) and (3).

Remark 2 "Hidden group structure" in a quantum group. Here the deformations are preferred, that is, the product on $\mathscr{D}_{t}(G)$ and on $\mathscr{A}_{t}(G)$ (resp. the coproduct on $\mathscr{C}_{t}^{\infty}(G)$ and on $\mathscr{H}_{t}(G)$ ) is not deformed and the basic structure is still the product on the group $G$. So this approach gives an interpretation of the Tannaka-Krein philosophy in the case of quantum groups: it has often been noticed that, in the generic case, Enite dimensional representations of a quantum group are (essentially) representations of its classical limit. So the algebras involved should be the same, which is justifed by the above mentioned rigidity result of Drinfeld. This shows that the initial classical 
group is still there, acting as a kind of "hidden variables" in this quantum group theory, which is exactly what we see in this quantum group theory. This fact was implicit in Drinfeld's work. The Tannaka-Krein interpretation of the twisting of quasi-Hopf algebras can be found in Majid (see e.g. [Ma92]). It was made explicit, within the framework exposed here, in [BFGP94].

\subsection{Unifcation of models and generalizations}

\subsubsection{Drinfeld models}

We call "Drinfeld model of quantum group" a deformation of $U \mathfrak{g}$ for $\mathfrak{g}$ simple, as given in [Dri87]. We have seen in the preceding section that from any Drinfeld model $\bigcup_{t} \mathfrak{g}$ of a quantum group (which can be generalized to any deformation of the Hopf algebra $U \mathfrak{g}$ ), we obtain a deformation of $\mathscr{D}(G)$ and $\mathscr{A}(G)$ that contains $\mathrm{U}_{t} \mathfrak{g}$ as a subHopf algebra. So $\mathscr{D}_{t}(G)$ and $\mathscr{A}_{t}(G)$ are quantum group models that describe Drinfeld models. By duality, $\mathscr{C}_{t}^{\infty}(G)$ and $\mathscr{H}_{t}(G)$ are "quantum group deformations" of $\mathscr{C}^{\infty}(G)$ and $\mathscr{H}(G)$. The deformed product on $\mathscr{H}(G)$ is the restriction of that on $\mathscr{C}^{\infty}(G)$. Furthermore, as we shall see, these deformations coincide with the usual "quantum algebras of functions". Let us look more in detail at $\mathscr{H}_{t}(G)$ :

\subsubsection{Faddeev-Reshetikhin-Takhtajan (FRT) models}

In [FRT88] quantized algebras of functions are de£ned in terms of generators and relations, the key relation being given by the star-triangle (Yang-Baxter) equation, $R(T \otimes \mathrm{ld})(\mathrm{Id} \otimes T)=(\mathrm{Id} \otimes T)(T \otimes \mathrm{Id}) R$, for a given $\mathrm{R}$-matrix $R \in \operatorname{End}(V \otimes V)$ and for $T \in \operatorname{End}(V), V$ being a fnite dimensional vector space.

As our deformations are given by a twist $P_{t}$, it is not surprising, from a structural point of view [Ma92] that, dually, we obtain in each case a Yang-Baxter relation and so a "FRT-type" quantized algebra of functions. Our Fréchet-topological context permits to write precisely such a construction for the infnite-dimensional Hopf algebras involved.

3.2.2.1. Linear case. If $G$ is semi-simple and linear, there exists $\pi$ a £nite dimensional representation of $G$ such that $\mathscr{H}(G) \simeq \mathbb{C}\left[\pi_{i j} ; 1 \leqslant i, j \leqslant N\right]$ where the $\pi_{i j}$ are the coeffcient functions of $\pi$. Denote by $\left(\mathscr{H}_{t}(G), *\right)$ the deformation of $\mathscr{H}(G)$ obtained in this way and by $T$ the matrix $\left[\pi_{i j}\right]$. Defne $T_{1}:=T \otimes I d$ and $T_{2}:=I d \otimes T$. Then we have

\section{Proposition 2 ([[BFGP94, BP96])}

1. $\left\{\pi_{i j} ; 1 \leqslant i, j \leqslant N\right\}$ is a topological generator system of the $\mathbb{C}[[t]]$-algebra $\mathscr{H}(G)_{t}$.

2. There exists an invertible $\mathscr{R} \in \mathscr{L}\left(V_{\pi} \otimes V_{\pi}\right)[[t]]$ such that $\mathscr{R} \cdot T_{1} * T_{2}=T_{2} * T_{1} \cdot \mathscr{R}$ (so $\mathscr{H}_{t}(G)$ is a "quantum algebra of functions" of type FRT).

3. We recover every quantum group given in [FRT88] by this construction.

\section{Sketch of proof.}

1. Perform a precise study of the deformed tensor product of representations.

2. Since the deformations $\mathscr{A}_{t}(G)$ are given by a twist $P_{t}, \mathscr{A}_{t}(G)$ is quasi-cocommutative, i.e. there exists $R \in(\mathscr{A}(G) \hat{\otimes} \mathscr{A}(G))[[t]]$ such that $\sigma \circ \Delta_{t}(a)=R \Delta_{t}(a) R^{-1}$ with $\sigma(a \otimes b)=b \otimes a$. Standard computations give the result.

3. We want to follow the way used in [Dri87] to link Drinfeld to FRT models. But the main point is that our deformations are obtained through a Drinfeld isomorphism. We therefore have to show:

- There exists a specifc Drinfeld isomorphism deforming the standard representation of $\mathfrak{g}$ into the representation of $\mathrm{U}_{t} \mathfrak{g}$ used in [Dri87].

- Two Drinfeld isomorphisms give equivalent deformations.

For instance, the FRT quantization of $S L(n)$ can be seen as a Hopf deformation of $\mathscr{H}(S U(n)$ ) (with non deformed coproduct). Moreover, this Hopf deformation extends to $\mathscr{C}^{\infty}(G)$. 


\section{Remark 3}

1. This proposition justifes the terminology "deformation", often employed but never justifed in these cases. See e.g. [GGS91] where it is shown that relations of type $\mathscr{R} T_{1} T_{2}=T_{2} T_{1} \mathscr{R}$ need not de£ne a deformation, even if $\mathscr{R}$ is Yang-Baxter.

2. Starting from Drinfeld models, our construction produces FRT models also for e.g. $G=\operatorname{Spin}(n)$ and for exceptional Lie groups. In addition, at least some multiparameter deformations [Res90] can be easily treated in this way [BFGP94].

\subsubsection{Non-linear case.}

Proposition 3 ([BP96]) If $G$ is semi-simple with £nite center, there exists a dense subalgebra of $\left(\mathscr{C}_{t}^{\infty}(G), *\right)$ generated by the coef£cient functions of a £nite number of (possibly in£nite dimensional) representations.

\subsubsection{Jimbo models}

These are models [Jim85] with generators $E_{i}^{ \pm}, K_{i}$ and $K_{i}^{-1}$. For $G=S U(2)$ BFP92] and $G=S L(2, \mathbb{C})$ [MZ96] we realize $\mathrm{U}_{q} \mathfrak{s l}(2)$ and $\mathrm{U}_{t} \mathfrak{s l}(2, \mathbb{C})$ as dense sub-Hopf algebras of $\mathscr{A}(G), \forall t \in \mathbb{C} \backslash 2 \pi \mathbb{Q}$ (with $q=e^{t}$ ). For $\mathfrak{s l}(2)$ this gives the original model of Jimbo [Jim85]. For the Lorentz algebra $\mathfrak{s l}(2, \mathbb{C})$ this unifes [MZ96] all the models proposed so far in the literature for a quantum Lorentz group. We obtain here convergent deformations (not only formal).

For $\mathfrak{s l}(2, \mathbb{C})$ it was $£$ rst proposed in [PW90] to consider the quantum double [Dri87] of $\bigcup_{q} \mathfrak{s u}(2)$ as $q$-deformed Lorentz group. It was known from [RSts90] that in such cases the double, as an algebra, is the tensor product of two copies of $\mathrm{U}_{t} \mathfrak{s u}(2)$. See also [OSWZ91, SWZ91], and [Ma93] for a dual version and another semi-direct product form.

\subsubsection{Deformation quantization}

From the main construction, using deformations of $U \mathfrak{g}$, we deduce the following general theorem:

Theorem 2 ([BP96]) Let $G$ be a semi-simple connected Lie group with a Poisson-Lie structure. There exists a deformation $\left(\mathscr{C}_{t}^{\infty}(G), *\right)$ of $\mathscr{C}^{\infty}(G)$ such that $*$ is a (differential) star product.

\section{Remark 4}

- When $\operatorname{Lie}(G)$ is the double of some Lie algebra, the same result holds.

- The fact that $*$ is differential comes from the twist $P_{t} \Delta_{0} P_{t}^{-1}, P_{t} \in(\mathrm{U} \mathfrak{g} \times \cup \mathfrak{g})[[t]]$.

- Since from any Drinfeld quantum group we obtain a star product, and since any FRT quantum group can be seen as a restriction of such a star product, we have showed that the data of a "semi-simple" quantum group is equivalent to the data of a star product on $\mathscr{C}^{\infty}(G)$ satisfying $\Delta(f * g)=\Delta(f) * \Delta(g)$. The functorial existence results of Etingof and Kazhdan [EK96] on the quantization of Lie bialgebras (see also [Enr02]) show that the latter is true also for "non semi-simple" quantum groups.

- Techniques similar to those indicated here can be applied to other $q$-algebras (more general quantum groups such as those in [Fro97] and more recent examples, Yangians, etc.). In particular those used in the case of the Jimbo models should be applicable to $q$-algebras de£ned by generators and relations. That direction of research has not yet been developed.

\section{Topological quantum double}

From now on we use the Sweedler notation for the coproducts Swe68: in a coalgebra $(H, \Delta), \Delta(x)=\sum_{(x)} x_{(1)} \otimes$ $x_{(2)}$ and, by coassociativity, $(\mathrm{Id} \otimes \Delta) \Delta(x)=(\Delta \otimes \mathrm{Id}) \Delta(x)=\sum_{(x)} x_{(1)} \otimes x_{(2)} \otimes x_{(3)}$.

In [Dri87] Drinfeld de£nes the quantum double of $\mathrm{U}_{t} \mathfrak{g}$ (see also [Sts94]). This can be adapted to the context of topological Hopf algebras [Bon94]. 


\subsection{De£nitions}

Let $A$ be $\mathscr{D}(G), \mathscr{A}(G), \mathscr{D}_{t}(G)$ or $\mathscr{A}_{t}(G)$. If $A=(A, \mu, \Delta, S)$ then $A^{*}=\left(A^{*},{ }^{t} \Delta,{ }^{t} \mu,{ }^{t} S\right)$. Defne $A^{0}=A^{* c o-o p}=$ $\left(A^{*},{ }^{t} \Delta,{ }^{t} \mu^{o p},{ }^{t} S^{o p}\right)$, where $\mu^{o p}(x \otimes y):=\mu(y \otimes x)$ and $S^{o p}$ is the antipode compatible with $\mu^{o p}$ and $\Delta$.

If we consider the vector space $A^{*} \otimes A$, Drinfeld [Dri87] de£nes the quantum double as follows :

i) $D(A) \simeq A^{0} \otimes A$ as coalgebras,

ii) $\left(f \otimes I d_{A}\right) \cdot\left(I d_{A^{0}} \otimes b\right)=f \otimes b$,

iii) $\left(I d_{A^{0}} \otimes e_{S}\right) \cdot\left(e^{t} \otimes I d_{A}\right)=\Delta_{s}^{k j n} \mu_{p l k}^{t} S_{n}^{\prime p}\left(e^{l} \otimes I d_{A}\right)\left(I d_{A^{0}} \otimes e_{j}\right)$, where $\left\{e_{s}\right\}$ is a basis of $A$ and $\left\{e^{t}\right\}$ the dual basis.

The Drinfeld double was expressed [Ma90] in a Sweedler form for dually paired Hopf algebras as an example of a theory of 'double smash products'. Adapting that formulation to our topological context we can now defne the double as:

Defnition 4 The double of $A, D(A)$, is the topological Hopf algebra $\left(A^{*} \bar{\otimes} A, \mu_{D},{ }^{t} \mu^{o p} \otimes \Delta,{ }^{t} S^{o p} \otimes S\right)$ with

$$
\begin{aligned}
\mu_{D}((f \otimes a) \otimes(g \otimes b)) & =\sum_{(a)} f<g, S^{o p}\left(a_{(3)}\right) ? a_{(1)}>\otimes a_{(2)} b \\
& =\sum_{(a)(g)}<g_{(1)}, a_{(1)}><{ }^{t} S^{o p}\left(g_{(3)}\right), a_{(3)}>f g_{(2)} \otimes a_{(2)} b
\end{aligned}
$$

where $<, \quad>$ denotes the pairing $A^{*} / A$, ,?" stands for a variable in A and $\bar{\otimes}$ is the completed inductive tensor product.

As topological vector spaces we have $D(A)=A^{*} \bar{\otimes} A$. Thus $D(A)^{*}=A \hat{\otimes} A^{*}$ and $D(A)^{* *}=D(A)$. So $D(A)$ is "almost self dual" (it is self dual up to a completion) and is rexexive.

\subsection{Extension theory}

- If $A$ is cocommutative then the product $\mu_{D}$ of $D(A)$ is the smash product $\vec{\mu}$ on $A^{0} \bar{\otimes} A$

$$
\vec{\mu}((f \otimes a) \otimes(g \otimes b))=\sum_{(a)} f\left(a_{(1)} \rightarrow g\right) \otimes a_{(2)} b
$$

where $\rightarrow$ denotes the coadjoint action of $A$ on $A^{0},<a \rightarrow f, b>=\sum_{(a)}<f, S\left(a_{(1)}\right) b a_{(2)}>$. This product is the "zero class" of an extension theory, de£ned by Sweedler [Swe68], classi£ed by a space of 2-cohomology $H_{s w}^{2}\left(A, A^{0}\right)$. The products are of the form, for $\tau$ a 2 -cocycle,

$$
\left.\overrightarrow{\mu_{\tau}}((f \otimes a) \otimes(g \otimes b))=\sum_{(a)(b)} f\left(a_{(1)} \rightarrow g\right) \tau\left(a_{(2)} \otimes b_{(2)}\right)\right) \otimes a_{(3)} b_{(2)} .
$$

- The coproduct of $D(A)$ is a smash coproduct for the trivial co-action. We can dualize the theory and, putting the two things together, we obtain an extension theory for bialgebras which is classifed by a cohomology space $H_{b i s w}^{2}\left(A^{0}, A\right)$.

Question : Are there other possible defnitions of the double as an extension of $A^{0}$ by $A$ ?

Answer : NO, for $A=\mathscr{D}(G)$ Bon94], because $H_{\text {bisw }}^{2}\left(\mathscr{D}(G), \mathscr{C}^{\infty}(G)\right)=\{0\}$.

\section{Crossed products and deformation quantization}

In this section we shall see that the Hopf algebra techniques presented in the preceding sections can be useful not only to understand quantum groups, but also to develop very nice formulas in deformation quantization itself.

In order to shed light on the general de£nition which follows, we return to the simplest case of deformation quantization: the Moyal product on $\mathbb{R}^{2}$. We look at $\mathbb{R}^{2}$ as $T^{*} \mathbb{R} \equiv \mathbb{R} \times \mathbb{R}^{*}$ and therefore can write $\mathscr{C}^{\infty}\left(\mathbb{R}^{2}\right) \simeq$ $\mathscr{C}^{\infty}(\mathbb{R}) \hat{\otimes} \mathscr{C}^{\infty}\left(\mathbb{R}^{*}\right)$. We consider frst two functions of a special kind in this algebra: $u(x)=u\left(x_{1}, x_{2}\right)=f\left(x_{1}\right) P\left(x_{2}\right)$ and $v(x)=v\left(x_{1}, x_{2}\right)=g\left(x_{1}\right) Q\left(x_{2}\right)$ where $f, g \in \mathscr{C}_{0}^{\infty}(\mathbb{R})$ and $P, Q$ are polynomials in $\operatorname{Pol}\left(\mathbb{R}^{*}\right) \simeq \mathrm{SR}$. We can then write is the usual coproduct on the symmetric algebra SR as $\left.\Delta(P)\left(x_{2}, y_{2}\right)=P\left(x_{2}+y_{2}\right) \stackrel{\text { notation }}{=} \sum_{(P)} P_{(1)}\left(x_{2}\right) P_{(2)}\left(y_{2}\right)\right)$. 
We now look at Formula (7) for the Moyal star product on $\mathbb{R}^{2}$ and perform on it some formal calculations (we do not discuss the convergence of the integrals involved). Up to a constant (depending on $\hbar$ ) we get:

$$
\begin{aligned}
(u * v)(x) & =\int_{\mathbb{R}^{2} \times \mathbb{R}^{2}} u(x+y) v(x+z) e^{-\frac{i}{\hbar} \Lambda^{-1}(y, z)} d y d z \\
& =\int_{\mathbb{R}^{2} \times \mathbb{R}^{2}} f\left(x_{1}+y_{1}\right) P\left(x_{2}+y_{2}\right) g\left(x_{1}+z_{1}\right) Q\left(x_{2}+z_{2}\right) e^{-\frac{i}{\hbar}\left(y_{1} z_{2}-y_{2} z_{1}\right)} d y_{1} d y_{2} d z_{1} d z_{2} \\
& =\int_{\mathbb{R}^{2}} f\left(x_{1}+y_{1}\right) Q\left(x_{2}+z_{2}\right) e^{-\frac{i}{\hbar} y_{1} z_{2}} d y_{1} d z_{2} \cdot \int_{\mathbb{R}^{2}} g\left(x_{1}+z_{1}\right) P\left(x_{2}+y_{2}\right) e^{\frac{i}{\hbar} y_{2} z_{1}} d y_{2} d z_{1} \\
& =\sum_{(P)(Q)}\left(\partial_{Q_{(1)}}^{+} f\right)\left(x_{1}\right) Q_{(2)}\left(x_{2}\right) \cdot\left(\partial_{P_{(1)}}^{-} g\right)\left(x_{1}\right) P_{(2)}\left(x_{2}\right) \quad \text { (up to a constant) }
\end{aligned}
$$

with $\partial_{Q_{(1)}}^{ \pm}=Q_{(1)}\left(\mp i \hbar \partial_{x_{1}}\right)$ (the same for $P$ ), since $F_{\hbar}^{\mp}\left(\alpha F_{\hbar}^{ \pm}(h)(\alpha)\right)(x)=\mp i \hbar \partial_{x} h(x)$ for $h \in \mathscr{C}_{0}^{\infty}(\mathbb{R})$ with $F_{\hbar}^{ \pm}(h)(\alpha)$ de£ned as $\int_{\mathbb{R}} h(x) e^{\mp \frac{i}{\hbar} x \alpha} d x$. This suggests the following small generalization of the smash product:

Defnition 5 Let $B$ be a cocommutative bialgebra and $C$ a B-bimodule algebra [i.e. $C$ is both a left B-module algebra and $a$ right $B$-module algebra such that $(a \rightarrow f)<b=a \rightarrow(f<b)]$. We de£ne the L-R smash product on $C \otimes B$ by

$$
(f \otimes a) \star(g \otimes b)=\sum_{(a)}\left(f<b_{(1)}\right)\left(a_{(1)} \rightarrow g\right) \otimes a_{(2)} b_{(2)} .
$$

Proposition 4 The L-R smash product is associative.

\subsection{Relation with usual deformation quantization}

Let $G$ be a Lie group, $T^{*} G$ its cotangent bundle, $\mathfrak{g}=\operatorname{Lie}(G)$. We have

$$
\mathscr{C}^{\infty}\left(T^{*} G\right) \simeq \mathscr{C}^{\infty}\left(G \times \mathfrak{g}^{*}\right) \simeq \mathscr{C}^{\infty}(G) \hat{\otimes} \mathscr{C}^{\infty}\left(\mathfrak{g}^{*}\right) \supset \mathscr{C}^{\infty}(G) \otimes \operatorname{Pol}\left(\mathfrak{g}^{*}\right) \simeq \mathscr{C}^{\infty}(G) \otimes \mathrm{Sg}
$$

We defne a deformation of $\mathscr{C}^{\infty}(G) \otimes \mathrm{S} \mathfrak{g}$ by a L-R smash product:

- We deform $S \mathfrak{g}$ by the "parametrized version" of $U \mathfrak{g}: \mathrm{U} \mathfrak{g}[[t]]=\frac{T \mathfrak{g}}{<x y-y x-t[x, y]\rangle}$. This is a Hopf algebra with $\Delta, \varepsilon$ and $S$ as for $U \mathfrak{g}$.

- Let $\left\{X_{i} ; i=1, \ldots, n\right\}$ be a basis of $\mathfrak{g}$ and $\vec{X}_{i}$ (resp. $\overleftarrow{X}_{i}$ ) be the left (resp. right) invariant vector £elds on $G$ associated with $X_{i}$. For $\lambda \in[0,1]$ we consider the following actions of $B=\cup \mathfrak{g}[[t]]$ on $C=\mathscr{C}^{\infty}(G)$ :

1. $\left(X_{i} \rightarrow f\right)(x)=t(\lambda-1)\left(\vec{X}_{i} \cdot f\right)(x)$

2. $\left(f<X_{i}\right)(x)=t \lambda\left(\overleftarrow{X}_{i} \cdot f\right)(x)$

Lemma 1 These actions de£ne on $\mathscr{C}^{\infty}(G)$ a B-bimodule algebra structure.

Defnition 6 We denote by $\star_{\lambda}$ the L-R smash product on $\mathscr{C}^{\infty}(G) \otimes \operatorname{Pol}\left(\mathfrak{g}^{*}\right)$ given by this B-bimodule algebra structure on $\mathscr{C}^{\infty}(G)$.

Proposition 5 For $G=\mathbb{R}^{n}, \star_{1 / 2}$ is the Moyal (Weyl ordered) star product, $\star_{0}$ is the standard ordered star product and in general $\star_{\lambda}$ is called $\lambda$-ordered star product on $\mathbb{R}^{2 n}[$ Pa99].

Remark 5 For a general Lie group $G, \star_{\lambda}$ gives in the generic case new deformation quantization formulas on $T^{*} G$. It would be interesting to study the properties of these $\star_{\lambda}$ for a noncommutative $G$ and their relations with the star products that are known. In particular $\star_{1 / 2}$ is formally different from the star product on $\mathscr{C}^{\infty}\left(T^{\star} G\right)$ given by S. Gutt in Gut83 but preliminary calculations seem to indicate that, in a neighborhood of the unit of $G$, they are equivalent by a symplectomorphism. 


\subsection{Application to the quantization of symmetric spaces}

Defnition 7 ([Bie95]) A symplectic symmetric space is a triple $(M, \omega, s)$, where $(M, \omega)$ is a smooth connected symplectic manifold and $s: M \times M \rightarrow M$ is a smooth map such that:

(i) for all $x$ in $M$, the partial map $s_{x}: M \rightarrow M: y \mapsto s_{x}(y):=s(x, y)$ is an involutive symplectic diffeomorphism of $(M, \omega)$ called the symmetry at $x$.

(ii) For all $x$ in $M, x$ is an isolated £xed point of $s_{x}$.

(iii) For all $x$ and $y$ in $M$, one has $s_{x} s_{y} s_{x}=s_{s_{x}(y)}$.

Two symplectic symmetric spaces $(M, \omega, s)$ and $\left(M^{\prime}, \omega^{\prime}, s^{\prime}\right)$ are isomorphic if there exists a symplectic diffeomorphism $\varphi:(M, \omega) \rightarrow\left(M^{\prime}, \omega^{\prime}\right)$ such that $\varphi s_{x}=s_{\varphi(x)}^{\prime} \varphi$.

De£nition 8 Let $(\mathfrak{g}, \sigma)$ be an involutive algebra, that is, $\mathfrak{g}$ is a £nite dimensional real Lie algebra and $\sigma$ is an involutive automorphism of $\mathfrak{g}$. Let $\Omega$ be a skewsymmetric bilinear form on $\mathfrak{g}$. Then the triple $(\mathfrak{g}, \sigma, \Omega)$ is called a symplectic triple if the following properties are satis£ed:

1. Let $\mathfrak{g}=\mathfrak{k} \oplus \mathfrak{p}$ where $\mathfrak{k}$ (resp. $\mathfrak{p})$ is the +1 (resp. - 1) eigenspace of $\sigma$. Then $[\mathfrak{p}, \mathfrak{p}]=\mathfrak{k}$ and the representation of $\mathfrak{k}$ on $\mathfrak{p}$, given by the adjoint action, is faithful.

2. $\Omega$ is a Chevalley 2-cocycle for the trivial representation of $\mathfrak{g}$ on $\mathbb{R}$ such that $\forall X \in \mathfrak{k}, i(X) \Omega=0$. Moreover, the restriction of $\Omega$ to $\mathfrak{p} \times \mathfrak{p}$ is nondegenerate.

The dimension of $\mathfrak{p}$ de£nes the dimension of the triple. Two such triples $\left(\mathfrak{g}_{i}, \sigma_{i}, \Omega_{i}\right)(i=1,2)$ are isomorphic if there exists a Lie algebra isomorphism $\psi: \mathfrak{g}_{1} \rightarrow \mathfrak{g}_{2}$ such that $\psi \circ \sigma_{1}=\sigma_{2} \circ \psi$ and $\psi^{*} \Omega_{2}=\Omega_{1}$.

Proposition 6 ([ [Bie95]) There is a bijective correspondence between the isomorphism classes of simply connected symplectic symmetric spaces $(M, \omega, s)$ and the isomorphism classes of symmetric triples $(\mathfrak{g}, \sigma, \Omega)$.

Defnition 9 A symplectic symmetric space $(M, \omega, s)$ is called an elementary solvable symplectic symmetric space if its associated triple $(\mathfrak{g}, \sigma, \Omega)$ is of the following type:

1. The Lie algebra $\mathfrak{g}$ is a split extension of Abelian Lie algebras $\mathfrak{a}$ and $\mathfrak{b}$ :

$$
\mathfrak{b} \longrightarrow \mathfrak{g} \leftrightarrows \mathfrak{a} .
$$

2. The automorphism $\sigma$ preserves the splitting $\mathfrak{g}=\mathfrak{b} \oplus \mathfrak{a}$.

3. There exists $\xi \in \mathfrak{k}^{*}$ such that $\Omega(X, Y)=\delta \xi=<\xi,[X, Y]_{\mathfrak{g}}>$ (Chevalley 2-coboundary).

For such an elementary solvable symplectic symmetric space there exists a global Darboux chart such that $(M, \omega) \simeq(\mathfrak{p}=\mathfrak{l} \oplus \mathfrak{a}, \Omega)[\operatorname{Bie} 00$. So we have

$$
\mathscr{C}^{\infty}(M) \simeq \mathscr{C}^{\infty}(\mathfrak{p}) \simeq \mathscr{C}^{\infty}(\mathfrak{l}) \hat{\otimes} \mathscr{C}^{\infty}(\mathfrak{a}) \simeq \mathscr{C}^{\infty}(\mathfrak{l}) \hat{\otimes} \mathscr{C}^{\infty}\left(\mathfrak{l}^{*}\right) \underset{a \simeq \mathfrak{l}^{*}}{\supset} \mathscr{C}^{\infty}(\mathfrak{l}) \otimes \operatorname{Pol}\left(\mathfrak{l}^{*}\right) \underset{\mathfrak{l} \text { abelian }}{\simeq} \mathscr{C}^{\infty}(\mathfrak{l}) \otimes U \mathfrak{l}
$$

One can now de£ne $\star_{1 / 2}$ (Moyal) on $\mathscr{C}^{\infty}(M) \simeq \mathscr{C}^{\infty}(\mathfrak{l} \oplus \mathfrak{a})$ or, using our preceding construction, on $\mathscr{C}^{\infty}(\mathfrak{l}) \otimes U \mathfrak{l}$. In order to have an invariant star product on $M$ under the action of $G$ (such that $\mathfrak{g}=\operatorname{Lie}(G)$ ) P. Bieliavsky [Bie00] defnes an integral transformation $S: \mathscr{C}^{\infty}(\mathfrak{l}) \rightarrow \mathscr{C}^{\infty}(\mathfrak{l})$ and then an invariant star product $\star_{S}$ by, for $T:=$ $S \otimes$ Id

$$
(f \otimes a) \star_{S}(g \otimes b):=T^{-1}\left(T(f \otimes a) \star_{1 / 2} T(g \otimes b)\right) .
$$

Let us defne $\quad f \bullet S g:=S^{-1}(S f . S g), \quad a \stackrel{S}{\rightarrow} f:=S^{-1}(a \rightarrow S f) \quad$ and $\quad f \stackrel{S}{\llcorner} a:=S^{-1}(S f<a)$.

Proposition $7([\overline{B B 02}]) \star_{S}$ is the L-R smash product of $\left(\mathscr{C}^{\infty}(\mathfrak{l}), \bullet_{S}\right)$ by $\mathrm{Ul}$ with the $\mathrm{Ul}$-bimodule structure given by $\stackrel{S}{\rightarrow}$ and $\stackrel{S}{-}$. 
Remark 6 Since we were dealing with quantum groups in the frst sections, we want to stress that the homogeneous (symmetric) spaces involved here are strictly different from those appearing in the quantum group approach of quantized homogeneous spaces [Dri93]. Indeed, in the latter, the spaces come from Poisson-Lie groups, so that the Poisson bracket has to be singular; therefore this bracket (and a fortiori a star product deforming this bracket) cannot be invariant (otherwise it would be zero everywhere). Here the Poisson brackets are invariant and regular.

Acknowledgments. This survey owes a lot to the insight shown by Moshé Flato in pushing forward the deformation quantization program, including in its aspects related to quantum groups where the inputs of Georges Pinczon and Murray Gerstenhaber were, as can be seen here, very important. Thanks are also due to the referee for a number of valuable comments.

\section{References}

[BFFLS] François Bayen, Moshé Flato, Christian Frønsdal, André Lichnerowicz and Daniel Sternheimer. Deformation theory and quantization I, II, Ann. Phys. (NY) (1978) 111, 61-110, 111-151.

[Bid96] Frédéric Bidegain. A candidate for a noncompact quantum group. Lett. Math. Phys., 36 (1996), 157167.

[BP96] Frédéric Bidegain and Georges Pinczon. A star-product approach to noncompact quantum groups. Lett. Math. Phys. 33 (1995), 231-240 (hep-th/9409054). Quantization of Poisson-Lie groups and applications. Comm. Math. Phys., 179 (1996), 295-332.

[Bie95] Pierre Bieliavsky. Espaces symétriques symplectiques. Ph. D. thesis, Université Libre de Bruxelles 1995.

[Bie00] Pierre Bieliavsky. Strict quantization of solvable symmetric spaces, math.QA/0011144 (2000).

[BB02] Pierre Bieliavsky and Philippe Bonneau. Quantization of solvable symmetric spaces II (work in progress).

[Bon92] Philippe Bonneau. Cohomology and associated deformations for not necessarily co-associative bialgebras. Lett. Math. Phys., 26 (1992), 277-283.

[Bon94] Philippe Bonneau. Topological quantum double. Rev. Math. Phys., 6 (1994), 305-318.

[BFGP94] Philippe Bonneau, Moshé Flato, Murray Gerstenhaber, and Georges Pinczon. The hidden group structure of quantum groups: strong duality, rigidity and preferred deformations. Comm. Math. Phys., 161 (1994), 125-156.

[BFP92] Philippe Bonneau, Moshé Flato, and Georges Pinczon. A natural and rigid model of quantum groups. Lett. Math. Phys., 25 (1992), 75-84.

[Co94] Alain Connes. Noncommutative Geometry, Academic Press, San Diego 1994.

[Co00] Alain Connes. Noncommutative geometry-year 2000. GAFA 2000 (Tel Aviv, 1999). Geom. Funct. Anal. (2000) Special Volume, Part II, 481-559 (math.QA/0011193).

[CDV02] Alain Connes and Michel Dubois-Violette. Noncommutative £nite-dimensional manifolds. I. Spherical manifolds and related examples. Comm. Math. Phys. 230 (2002), 539-579 (math.QA/0107070).

[CFS92] Alain Connes, Moshé Flato and Daniel Sternheimer. Closed star-products and cyclic cohomology, Lett. Math. Phys. 24 (1992), 1-12.

[CK99] Alain Connes and Dirk Kreimer. Lessons from Quantum Field Theory — Hopf Algebras and Spacetime Geometries. Lett. Math. Phys. 48 (1999), 85-96.

[CM03] Alain Connes and Henri Moscovici. Modular Hecke Algebras and their Hopf Symmetry, math.QA/0301089. Rankin-Cohen Brackets and the Hopf Algebra of Transverse Geometry, math.QA/0304316. 
[DS02] Giuseppe Dito and Daniel Sternheimer. Deformation Quantization: Genesis, Developments and Metamorphoses, in Deformation quantization (G.Halbout ed.), IRMA Lectures in Math. Theoret. Phys. 1, pp. 9-54 . Walter de Gruyter, Berlin 2002 (math. QA/0201168).

[DN01] Michael R. Douglas and Nikita A. Nekrasov. Noncommutative Field Theory. Rev.Mod.Phys. 73 (2001), 977-1029 (hep-th/0106048).

[Dri87] Vladimir G. Drinfel'd. Quantum groups, in Proceedings of the International Congress of Mathematicians, Vol. 1-2 (Berkeley, Calif., 1986), pp. 798-820, Amer. Math. Soc. Providence, RI 1987.

[Dri89] Vladimir G. Drinfel'd. Almost cocommutative Hopf algebras. Algebra i Analiz, 1 (1989), 30-46.

[Dri93] Vladimir G. Drinfel'd. On Poisson homogeneous spaces of Poisson-Lie groups. Teoret. Mat. Fiz., 95 (1993), 226-227; translation in Theoret. and Math. Phys., 95 (1993), 524-525.

[Enr02] Benjamin Enriquez. A cohomological construction of quantization functors of Lie bialgebras. math.QA/0212325 (2002).

[EK96] Pavel Etingof and David Kazhdan. Quantization of Lie bialgebras I. Selecta Math. (N.S.), 2 (1996), 1-41; II, III ibid. 4 (1998), 213-231, 233-269; IV, V ibid. 6 (2000), 79-104, 105-130; Quantization of Poisson algebraic groups and Poisson homogeneous spaces, in Symétries quantiques (Les Houches, 1995), 935-946, North-Holland, Amsterdam 1998.

[FRT88] Ludwig D. Faddeev, Nicolai Yu. Reshetikhin, and Leon A. Takhtajan. Quantization of Lie groups and Lie algebras, in Algebraic analysis, Vol. I, pp. 129-139. Academic Press, Boston, MA 1988.

[Fla82] Moshé Flato. Deformation view of physical theories, Czechoslovak J. Phys. B32 (1982), 472-475.

[Fro97] Christian Frønsdal. Generalization and exact deformations of quantum groups. Publ. Res. Inst. Math. Sci. 33 (1997), 91-149.

[Ger64] Murray Gerstenhaber. On the deformation of rings and algebras, Ann. Math. 79 (1964), 59-103; and (IV), ibid. 99 (1974), 257-276.

[GGS91] Murray Gerstenhaber, Anthony Giaquinto and Samuel D. Schack. Quantum symmetry, in Lect. Notes in Math. 1510, 9-46, Springer 1991.

[GS88] Murray Gerstenhaber and Samuel D. Schack. Algebraic cohomology and deformation theory, in Deformation Theory of Algebras and Structures and Applications (M. Hazewinkel and M. Gerstenhaber Eds.), NATO ASI Ser. C 247, 11-264, Kluwer Acad. Publ., Dordrecht 1988.

[GS90] Murray Gerstenhaber and Samuel D. Schack. Bialgebra cohomology, deformations, and quantum groups. Proc. Natl. Acad. Sci. USA, 87 (1990) 478-481.

[Gre46] Hip J. Groenewold. On the principles of elementary quantum mechanics. Physica 12 (1946), 405-460.

[Grt55] Alexander Grothendieck. Produits tensoriels topologiques et espaces nucléaires. Mem. Amer. Math. Soc., No 16 (140p.), 1955.

[Gut83] Simone Gutt. An explicit *-product on the cotangent bundle of a Lie group. Lett. Math. Phys., 7 (1983), 249-258.

[Jim85] Michio Jimbo. A $q$-difference algebra of $\mathscr{U}(\mathfrak{g})$ and the Yang-Baxter equation. Lett. Math. Phys. 10 (1985), 63-69.

[Ko01] Maxim Kontsevich. Deformation quantization of algebraic varieties. in EuroConférence Moshé Flato 2000, Part III (Dijon), Lett. Math. Phys. 56 (2001), 271-294.

[KZ01] Maxim Kontsevich and Don Zagier. Periods, in Mathematics unlimited-2001 and beyond, 771-808, Springer, Berlin 2001.

[Ma90] Shahn Majid. Physics for algebraists: noncommutative and noncocommutative Hopf algebras by a bicrossproduct construction. J. Algebra 130 (1968), 17-64. 
[Ma92] Shahn Majid. Tannaka-Krein theorem for quasi-Hopf algebras and other results, in Deformation theory and quantum groups with applications to mathematical physics (Amherst, MA, 1990), Contemp. Math., 134 (1992), 219-232.

[Ma93] Shahn Majid. Braided matrix structure of the Sklyanin algebra and of the quantum Lorentz group. Comm. Math. Phys. 156 (1993), 607-638. See also: A quantum groups primer, London Mathematical Society Lecture Note Series 292, x+169 pp., Cambridge University Press, Cambridge, 2002.

[MZ96] Christiane Martin and Mohamed Zouagui. A noncommutative Hopf structure on $\mathscr{C}^{\infty}[S L(2, \mathbf{C})]$ as a quantum Lorentz group. J. Math. Phys., 37 (1996), 3611-3629.

[Moy49] Jose E. Moyal. Quantum mechanics as a statistical theory, Proc. Cambridge Phil. Soc. 45 (1949), 99124.

[OSWZ91] Oleg Ogievetsky, William B. Schmidke, Julius Wess, and Bruno Zumino. Six generator $q$-deformed Lorentz algebra. Lett. Math. Phys. 23 (1991), 233-240.

[PW90] Piotr Podleś and Stanis“aw L. Woronowicz. Quantum deformation of Lorentz group. Comm. Math. Phys. 130 (1990), 381-431.

[Pa99] Markus J. Paaum. Deformation quantization on cotangent bundles, in Coherent states, differential and quantum geometry (Bia“owieła 1997), Rep. Math. Phys., 43 (1999), 291-297.

[Res90] Nicolai Yu. Reshetikhin. Multiparameter quantum groups and twisted quasitriangular Hopf algebras. Lett. Math. Phys. 20 (1990), 331-335.

[RSts90] Nicolai Yu. Reshetikhin and Michael A. Semenov-Tian-Shansky. Quantum $R$-matrices and factorization problems. J. Geom. Phys. 5(4) (1989), 533-550.

[SWZ91] William B. Schmidke, Julius Wess and Bruno Zumino. A q-deformed Lorentz algebra. Z. Phys. C 52 (1991), 471-476.

[Sts94] Michael A. Semenov-Tian-Shansky. Poisson Lie groups, quantum duality principle, and the quantum double, in Mathematical aspects of conformal and topological £eld theories and quantum groups (South Hadley, MA, 1992), Contemp. Math. 175, 219-248. Amer. Math. Soc., Providence, RI 1994.

[Swe68] Moss E. Sweedler. Cohomology of algebras over Hopf algebras. Trans. Am. Math. Soc. 133 (1968), 205-239.

[Tré67] François Tréves. Topological vector spaces, distributions and kernels, xvi+624 pp., Academic Press, New York-London 1967.

[Wey31] Hermann Weyl. The theory of groups and quantum mechanics, Dover, New-York 1931. Gruppentheorie und Quantenmechanik, Reprint of the second edition [Hirzel, Leipzig 1931], xi+366 pp. Wissenschaftliche Buchgesellschaft, Darmstadt 1977.

[Wig32] Eugene P. Wigner. Quantum corrections for thermodynamic equilibrium, Phys. Rev. 40 (1932), 749_ 759. 